\title{
Evolución de la educación en valores y su proyección social en la escuela inclusiva
}

\section{The evolution of education in values and social projection in inclusive schools}

\author{
Rosa $M^{a}$ Santamaría Conde ${ }^{1}$ \\ rsantamaria@ubu.es \\ Miguel Corbí Santamaría \\ mcorbi@ubu.es \\ Universidad de Burgos, España
}

\section{Resumen:}

En el presente artículo se realiza un análisis crítico-descriptivo de la evolución que ha experimentado la educación en valores dentro sistema educativo español. Se analiza dicha educación a través de los temas transversales y el desarrollo de las competencias que contempla la legislación vigente, todo ello en el marco de la escuela inclusiva como una superación de la escuela integradora. Asimismo, se describe la proyección social que tiene la educación en valores que se desarroIla en el ámbito escolar, apostando por el fomento de una buena convivencia y una resolución pacífica del conflicto, todo ello con la intención de alcanzar una formación integral y armónica a nivel personal y social del niño y adolescente.

\section{Palabras clave:}

Sociedad; escuela inclusiva; educación en valores; temas transversales; competencias.

\begin{abstract}
:
The present paper carries out a criticaldescriptive analysis of the evolution of education in values experienced in the Spanish education system. This type of education is looked at through transversal topics of discussion and the development of the competences included in the current legislation, all within the framework of inclusive schools as an alternative to the integrative school. Also, the social projection of education in values present in the schools is described, advocating for the promotion of proper coexistence and peaceful conflict resolution, all within an integral and harmonious development of children and adolescents at a personal and social level.
\end{abstract}

\section{Key words:}

Society; inclusive school; education in values; transversal themes; competences.

1 Dirección para correspondencia (correspondence address):

Rosa M $M^{a}$ Santamaría Conde. Universidad de Burgos. Departamento de Ciencias de la Educación. Facultad de Educación. C/Villadiego, 1. 09001 Burgos (España). 


\section{Résumé :}

Cet article réalise une analyse critico-descriptive sur l'évolution expérimentée par l'éducation aux valeurs dans le système éducatif espagnol. Cette éducation est analysée à travers les questions transversales et le développement des compétences prévues par la législation en vigueur, tout cela dans le cadre de l'école inclusive comme un dépassement de l'école intégratrice. On décrit en même temps la projection sociale de l'éducation aux valeurs qui se déroulent dans les écoles, en pariant sur la promotion d'une bonne coexistence et d'une résolution pacifique des conflits, tout cela dans le but d'obtenir une formation de niveau global et harmonieuse au niveau personnel et social de l'enfant et de l'adolescent.

\section{Mots clés :}

Société; école inclusive; l'éducation aux valeurs; thèmes transversaux; compétences.

Fecha de recepción: 18-11-2016

Fecha de aceptación: 06-02-2017

\section{Introducción}

A lo largo de los últimos años mucho ha cambiado la escuela en lo que se refiere a sus destinatarios, encontrándonos grupos de alumnos cada vez más heterogéneos, no sólo en cuanto a sus capacidades sino también en lo que se refiere a sus necesidades e intereses. Si en los años ochenta se hablaba de la importancia de integrar en los centros educativos ordinarios a los alumnos con cualquier tipo de discapacidad, hoy se hace un especial énfasis en favorecer la inclusión de todos y cada uno de los niños y niñas que están escolarizados sobre todo en las etapas de Educación Infantil, Primaria y Secundaria Obligatoria.

El término de inclusión es mucho más amplio que el de integración, ya que supone que no sólo el alumno, con sus características particulares, debe estar integrado en el ámbito educativo, sino que hay que garantizar la igualdad de oportunidades de todos ellos dentro de un contexto social, de ahí que al hablar de la escuela inclusiva deba entenderse como una proyección más allá de lo estrictamente escolar. Muntaner, Rosselló y De la Iglesia (2016, p. 35), entienden que

La educación inclusiva, al igual que cualquier otro modelo educativo, tiene en los valores y en las creencias los fundamentos que la sostienen y se explican por una teoría, es decir, por unos referentes que justifican las decisiones y las prácticas realizadas. 
Ante esta nueva concepción de los centros educativos, la educación en valores adquiere un protagonismo especial y relevante dentro de la inclusión. Hoy en día el hecho de que en nuestras escuelas haya alumnos de diferencias etnias y distinta procedencia supone que en ocasiones dicha multiculturalidad implique una escala de valores diversa; de ahí que la necesidad de fomentar el respecto, el diálogo, la tolerancia y la libertad se convierta en el principio rector de toda actividad educativa con una clara proyección en el ámbito social. A este respecto, el estudio de Buxarrais (2003, p. 81) entiende que "educar en valores significa encontrar espacios de reflexión tanto individual como colectiva, para que el alumnado sea capaz de elaborar de forma racional y autónoma los principios de valor [...]". Por otro lado, Bisquerra (2011) y López Melero (2012) apuestan por la necesidad de favorecer una educación en valores en el marco de la escuela inclusiva, siendo una responsabilidad compartida por toda la comunidad educativa, e inclusive por la sociedad.

El actual sistema educativo apuesta por una educación en valores que se desarrolla fundamentalmente a través del desarrollo de los temas transversales y de las competencias, siendo conscientes que no siempre resulta fácil Ilevarlo a la práctica. A este respecto, Yus Ramos (2002, p. 42) destaca que

\begin{abstract}
Nuestra cultura ha estado tan fuertemente influida por la organización del conocimiento en torno a disciplinas que nos cuesta trabajo entender que las disciplinas no son un fin, sino un medio para comprender y transformar la realidad. Por ello, cuando algo entra en la escuela, automáticamente tendemos a «asignaturalizarlo», buscando con ello la relevancia o fortaleza de las poderosas asignaturas clásicas.
\end{abstract}

Por todo ello, una verdadera educación en valores, dentro de la escuela en general y de la inclusiva en particular, debe intentar que la persona aprenda a ser, aprenda a convivir y aprenda a participar, en esta línea es necesario trabajar conjuntamente escuela y familia y que tenga una proyección social que supere el ámbito estrictamente escolar.

Así pues, a lo largo de este artículo se pretende analizar el desarrollo de la educación en valores que debe guiar el proceso de enseñanzaaprendizaje en la escuela inclusiva, tomando como referencia sobre todo la legislación que hay al respecto. 


\section{La educación en valores en el Sistema Educativo Español}

Como ya es sabido, la educación obligatoria tiene como objetivo prioritario el desarrollo integral de todas aquellas capacidades y destrezas en el alumnado que se consideran necesarias para desenvolverse como ciudadanos con plenos derechos y deberes en la sociedad en la que viven; es decir, la educación integral supone atender no sólo a las capacidades intelectuales, sino también a las capacidades afectivas, motrices, de relación interpersonal y de inserción y actuación social. A este respecto, sobre todo a partir la promulgación de la Ley Orgánica General del Sistema Educativo (LOGSE, 1990), hay ciertas cuestiones que empiezan a formar parte del currículo explícito, que son aspectos que la sociedad actual reclama como una atención prioritaria, tales como la violencia, la escasa presencia de valores éticos básicos, las discriminaciones y desigualdades sociales, el consumismo, la degradación del medio ambiente, los hábitos de vida que atentan contra una existencia saludable, etc.

La educación debe posibilitar que el alumnado llegue a entender estos problemas cruciales y a elaborar un juicio crítico respecto a ellos, siendo capaces de adoptar actitudes y comportamientos basados en valores racionales y libremente asumidos; en definitiva, se debe potenciar una educación en valores que contribuya a la formación como personas capaces de desarrollarse personal y socialmente. Consecuencia de esta reflexión es la que llevó al entonces Ministerio de Educación y Ciencia a introducir en los Decretos del Currículo de Educación Infantil, Primaria y Secundaria Obligatoria un tipo de enseñanzas que respondiesen a estos problemas sociales y que se denominan temas transversales. Bajo este concepto se agruparon una serie de contenidos que formaban parte de un currículo explícito y que debían reflejarse en los respectivos proyectos curriculares y educativos de centro, y en las programaciones de aula. Estos temas eran: educación moral y cívica, educación para la paz, educación para la igualdad de oportunidades de ambos sexos, educación para la salud, educación sexual, educación ambiental, educación del consumidor, educación vial. En definitiva, se apostaba por unas estrategias de educación en valores que contribuyesen "al desarrollo de la autonomía personal y moral, y que capaciten para la participación social y responsable" (Ministerio de Educación y Ciencia, 1993, p. 33).

La incorporación de los aspectos sociales a través de los temas transversales supuso en la práctica la asunción por parte del sistema educati- 
vo de una función ético-moral de la educación, desde una concepción humanista que complementa la científica; es decir,

Los temas transversales no son un añadido, ni algo que se plantea en paralelo o marginalmente al currículo y que hay que ir integrando, punto por punto, en el diseño de las Áreas, sino que constituyen un proyecto global de valores, o proyecto de humanización, que no sólo es previo a las Áreas, sino que las fundamenta y las redimensiona en su totalidad, redimensionando, a su vez, todo el Proyecto Educativo de Centro (González Lucini, 1994, p. 135).

Una enseñanza dirigida a desarrollar en el alumnado una formación integral, implicando tanto la dimensión disciplinar como la dimensión ético-moral, supone lógicamente una apuesta por una educación en valores, Ilegando a entender determinados conceptos, actuando conforme a ciertas maneras de saber hacer, y desarrollando un determinado sistema de valores que podría resumirse en una actitud democrática, responsable, tolerante, que favorezca la participación activa y solidaria y asegurando niveles cada vez más altos de libertad, igualdad y justicia social. A este respecto, el desarrollo de los temas transversales intentaba garantizar una educación en valores en el amplio sentido de la palabra, que no podía reducirse a actuaciones esporádicas o puntuales, sino que debía formar parte de un proyecto educativo asumido por toda la comunidad escolar. Por ello, el modelo ético que se promovía desde los temas transversales tenía que ser asumido por el conjunto de la institución educativa para que finalmente cada alumno lo interiorice y haga suyo, dado que estos aprendizajes se producen a través de la práctica personal y diaria.

Los temas transversales requirieron para su desarrollo una colaboración con el medio y, por tanto, mantener una relación de la escuela con el entorno. En primer lugar, por la estrecha vinculación con la realidad social, y en segundo lugar, porque existen numerosas instituciones y organismos nacionales e internacionales que trabajan en ello, y que se plantean colaborar en los centros escolares desde un convencimiento claro de que la educación es un camino básico para conseguir mejoras sociales. Asimismo, implicaba la incorporación de nuevas enseñanzas o modelos educativos.

Reconocida por Ley la autonomía organizativa y pedagógica (junto con la económica) de los centros educativos, la elaboración de los do- 
cumentos institucionales cobra una especial relevancia. La vinculación o relación que existe, o que debe existir, entre ética y educación, es uno de los principios pedagógicos más importantes y más innovadores de nuestra escuela y de la intervención del profesorado en ella, y por ello debe exponerse clara y explícitamente en los proyectos educativos de centro y en los distintos documentos que se derivan de éstos (concreción del currículo, programación general anual, plan de convivencia, etc.).

En la Reforma educativa de 1990 se planteaban tres principios esenciales:

- Se suprimen los programas y las clases de Ética como alternativa a los de Religión y Moral Católica. Todos los alumnos, en una sociedad pluralista y democrática, tienen el derecho y la necesidad de recibir una formación ética al margen de sus creencias religiosas, y por ello, deben ser educados con libertad, en un sistema de valores que les ayude a saber vivir con ellos mismos y con los demás.

- Se concibe la educación ética como una educación en valores y actitudes, una educación no planteada de forma esporádica, sino como algo que debe integrarse e impregnar toda la actividad escolar, todos los procesos de aprendizaje y todas las áreas. De ahí su carácter de transversalidad y su presencia en todas las etapas, ciclos y bloques temáticos de todas las áreas, bien en los contenidos actitudinales, bien como unos contenidos de especial relevancia, como en el caso concreto de los calificados como temas transversales.

- En la Educación Secundaria Obligatoria, y más en concreto dentro del segundo ciclo, en el área de Ciencias Sociales, Geografía e Historia, se establecen unos contenidos, obligatorios para todos los alumnos, incluidos bajo el epígrafe: la vida moral y la reflexión ética; contenidos que deben impartirse y organizarse como una materia, sin perjuicio de los restantes contenidos del área.

Con la promulgación de la Ley Orgánica de Educación (LOE, 2006) los temas transversales como tal no aparecen de forma explícita, pero sí hace alusión a la educación en valores en los siguientes términos:

- En el Preámbulo se señala que "esta educación [educación para la ciudadanía], cuyos contenidos no pueden considerarse en ningún caso alternativos o sustitutorios de la enseñanza religiosa, no entra en contradicción con la práctica democrática que debe inspirar el 
conjunto de la vida escolar y que ha de desarrollarse como parte de la educación en valores con carácter transversal a todas las actividades escolares".

- Entre los principios de la educación se destaca: "la transmisión y puesta en práctica de valores que favorezcan la libertad personal, la responsabilidad, la ciudadanía democrática, la solidaridad, la tolerancia, la igualdad, el respeto y la justicia, así como que ayuden a superar cualquier tipo de discriminación" (art. 1.c). Y añade al respecto: "la orientación educativa y profesional de los estudiantes, como medio necesario para el logro de una formación personalizada, que propicie una educación integral en conocimientos, destrezas y valores" (art. 1.f).

- Entre los fines de la educación a conseguir destacar: "la formación para la paz, el respeto a los derechos humanos, la vida en común, la cohesión social, la cooperación y solidaridad entre los pueblos así como la adquisición de valores que propicien el respeto hacia los seres vivos y el medio ambiente, en particular al valor de los espacios forestales y el desarrollo sostenible" (art. 2.e)

- Al hacer alusión a los principios pedagógicos de la Educación Primaria se establece: "sin perjuicio de su tratamiento específico en algunas áreas de la etapa, la comprensión lectora, la expresión oral y escrita, la comunicación audiovisual, las tecnologías de la información y la comunicación y la educación en valores se trabajarán en todas las áreas" (art. 19.2). Este mismo tratamiento se incluye también en Educación Secundaria Obligatoria (arts. 24.7 y 25.5).

Como puede constatarse, actualmente la educación en valores se plantea con un tratamiento transversal, aplicable a todo el ámbito educativo y debería también tener su proyección en el ámbito social en general. Asimismo, conviene señalar también lo regulado en el Real Decreto 126/2014, por el que se establece el currículo básico de la Educación Primaria (a partir de la Ley Orgánica para la Mejora de la Calidad Educativa, LOMCE, 2013), que en su artículo 10 hace alusión explícita a los "elementos transversales", destacando entre otros los siguientes aspectos:

- La comprensión lectora, la expresión oral y escrita, la comunicación audiovisual, las Tecnologías de la Información y la Comuni- 
cación, el emprendimiento y la educación cívica y constitucional se trabajarán en todas las asignaturas.

- Las Administraciones educativas fomentarán el desarrollo de los valores que fomenten la igualdad efectiva entre hombres y mujeres y la prevención de la violencia de género, y de los valores inherentes al principio de igualdad de trato y no discriminación por cualquier condición o circunstancia personal o social.

- Las Administraciones educativas fomentarán el aprendizaje de la prevención y resolución pacífica de conflictos en todos los ámbitos de la vida personal, familiar y social, así como de los valores que sustentan la libertad, la justicia, la igualdad, el pluralismo político, la paz, la democracia, el respeto a los derechos humanos y el rechazo a la violencia terrorista, la pluralidad, el respeto al Estado de derecho, el respeto y consideración a las víctimas del terrorismo y la prevención del terrorismo y de cualquier tipo de violencia.

- En los currículos de Primaria se incorporarán elementos curriculares relacionados con: la prevención de la violencia de género, de la violencia terrorista y de cualquier forma de violencia, racismo o xenofobia, el desarrollo sostenible y el medio ambiente, los riesgos de explotación y abuso sexual, las situaciones de riesgo derivadas de la utilización de las Tecnologías de la Información y la Comunicación, así como la protección ante emergencias y catástrofes.

- Las Administraciones educativas fomentarán las medidas para que el alumnado participe en actividades que le permita afianzar el espíritu emprendedor y la iniciativa empresarial a partir de aptitudes como la creatividad, la autonomía, la iniciativa, el trabajo en equipo, la confianza en uno mismo y el sentido crítico. También adoptarán medidas para que la actividad física y la dieta equilibrada formen parte del comportamiento infantil, garantizando un desarrollo adecuado para favorecer una vida activa, saludable y autónoma, así como promover acciones para la mejora de la convivencia y la prevención de los accidentes de tráfico.

A todo ello, tampoco se puede obviar que en este artículo 10 se hace alusión de un modo explícito a la atención al alumnado con discapacidad en los siguientes términos: 
Las Administraciones educativas fomentarán la calidad, equidad e inclusión educativa de las personas con discapacidad, la igualdad de oportunidades y no discriminación por razón de discapacidad, medidas de flexibilización y alternativas metodológicas, adaptaciones curriculares, accesibilidad universal, diseño para todos, atención a la diversidad y todas aquellas medidas que sean necesarias para conseguir que el alumnado con discapacidad pueda acceder a una educación educativa de calidad en igualdad de oportunidades (art. 10.2).

Asimismo, en el Real Decreto 1105/2014, de 26 de diciembre, por el que se establece el currículo básico de la Educación Secundaria Obligatoria y del Bachillerato, se regula como uno de los objetivos concretos de la Educación Secundaria Obligatoria:

Asumir responsablemente sus deberes, conocer y ejercer sus derechos en el respeto a los demás, practicar la tolerancia, la cooperación y la solidaridad entre las personas y grupos, ejercitarse en el diálogo afianzando los derechos humanos y la igualdad de trato y de oportunidades entre mujeres y hombres, como valores comunes de una sociedad plural y prepararse para el ejercicio de la ciudadanía democrática (art. 11.a).

A este respecto, es evidente que la escuela inclusiva adquiere un protagonismo total en el desarrollo de una educación en valores dentro de esta transversalidad que la Ley promueve. En este sentido, Del Carmen (2004, p. 49) entiende que la atención a la diversidad, y por ende a la inclusión, es una cuestión de valores, ya que "para que la escuela pueda afrontar con éxito la gran diversidad existente en las aulas es importante avanzar hacia una nueva manera de entender y valorar la situación y el papel que la escuela tiene". Se puede decir que, frente a la cultura de la homogeneidad, la escuela y por supuesto también la sociedad, debe apostar por la cultura de la diversidad dentro del marco inclusivo, y a partir de aquí fomentar los valores que lo hagan posible.

Sin embargo, no se debe olvidar que la educación en valores tiene demasiadas aristas (figura 1), no es algo totalmente lineal; es decir, en la medida que la sociedad evoluciona y al mismo tiempo teniendo en cuenta las experiencias personales de cada uno se produce una reestructuración de valores; por otro lado, los valores como tal tampoco pueden abordarse en términos absolutos. 


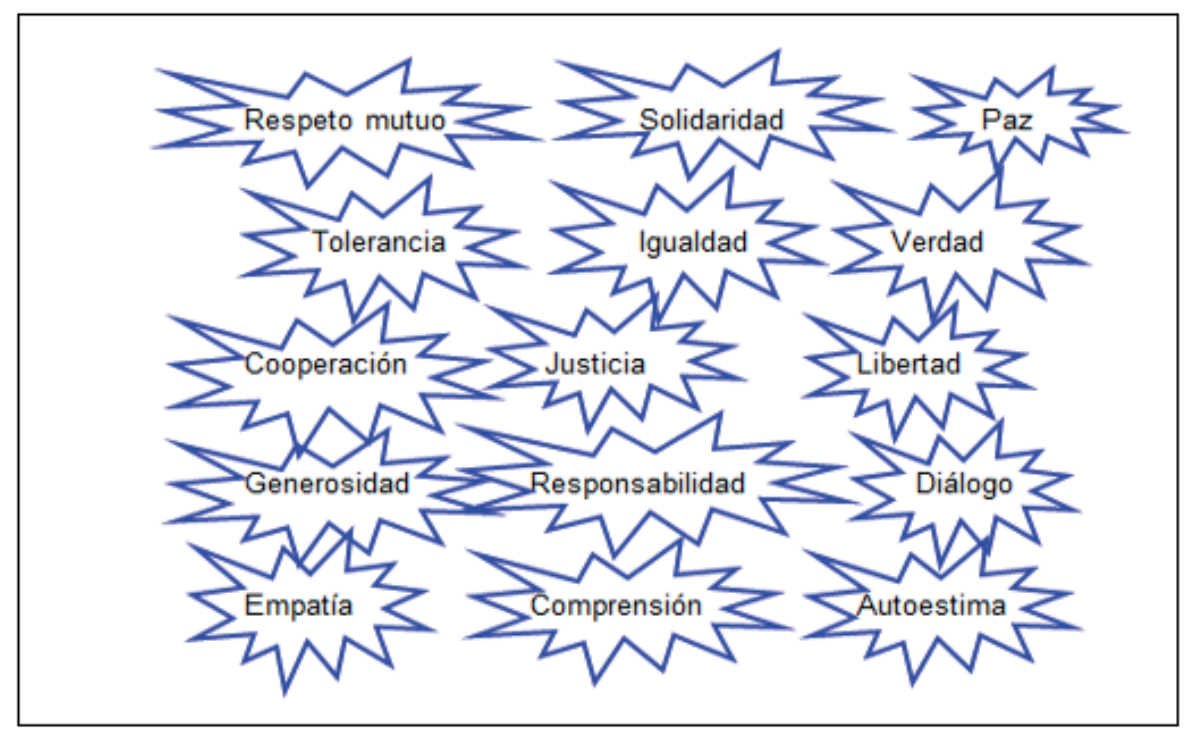

Figura 1. La complejidad de los valores. Elaboración propia.

Ante este panorama, es evidente que la educación en valores no es una tarea fácil dentro de la escuela inclusiva, teniendo en cuenta además que la sociedad, en general, y la familia, en particular, tienen una gran influencia en el niño y adolescente. Como expone Rosales (2015, p. 30), "es evidente la necesidad de establecer puentes de comunicación y de coordinar los esfuerzos de todos los agentes educativos", de ahí que sea necesario que cada uno asuma sus responsabilidades (escuela, familia, sociedad), pero trabajen de modo colaborativo, asumiendo un esfuerzo compartido. Por su parte, Domínguez Chillón (1999) al referirse a la educación en valores en Educación Infantil, concreta una serie de requisitos que facilitan el desarrollo de los valores y que podrían aplicarse a toda la educación básica: un ambiente vitalizante tanto físico como psíquico, una reflexión y sentido crítico que propicie el respeto hacia los demás, la apuesta por la significatividad de los aprendizajes que tenga en cuenta también los aspectos afectivos, la sensibilidad del profesorado hacia el desarrollo de los valores, la estrecha colaboración con las familias, y por último, la autoestima y la colaboración que favorezca el diálogo, el sentido de responsabilidad, la negociación, etc.

A la vista de todo ello, la educación en valores dentro del actual sistema educativo español es algo que compete a todos como miembros de la sociedad, no siendo algo exclusivo de la escuela. 


\subsection{La educación en competencias y su relación con la educación en valores}

La Ley Orgánica de Ordenación General del Sistema Educativo (LOGSE, 1990) contemplaba la posibilidad de que los contenidos fuesen de tipo conceptual, procedimental y actitudinal. Precisamente los contenidos actitudinales junto con los temas transversales contribuían a la educación en valores en las aulas, suponiendo un importante avance en lo que se refiere a dicha educación que hasta entonces había permanecido en un segundo nivel. La Ley Orgánica de Educación (LOE, 2006) no contempla dicha separación, y en su artículo 6 entiende por currículo "el conjunto de objetivos, competencias básicas, contenidos, métodos pedagógicos y criterios de evaluación". Asimismo, en el desarrollo de los Reales Decretos de enseñanzas mínimas de Educación Primaria y Secundaria Obligatoria se explicita que "la incorporación de competencias básicas al currículo permite poner el acento en aquellos aprendizajes que se consideran imprescindibles, desde un planteamiento integrador y orientado a la aplicación de los saberes adquiridos". En el marco de la propuesta realizada por la Unión Europea se determinan cuáles son las competencias básicas que se deben desarrollar en la escolaridad básica y obligatoria, concretándose en las siguientes:

- Competencia en comunicación lingüística.

- Competencia matemática.

- Competencia en el conocimiento y la interacción con el mundo físico.

- Tratamiento de la información y competencia digital.

- Competencia social y ciudadana.

- Competencia cultural y artística.

- Competencia para aprender a aprender.

- Autonomía e iniciativa personal.

Con la promulgación de la Ley Orgánica 8/2013, de 9 de diciembre, para la Mejora de la Calidad Educativa (LOMCE, 2013) se inciden en las competencias anteriormente reguladas con algunas matizaciones:

- Comunicación lingüística.

- Competencia matemática y competencias básicas en ciencia y tecnología.

- Competencia digital. 
- Aprender a aprender.

- Competencias sociales y cívicas.

- Sentido de iniciativa y espíritu emprendedor.

- Conciencia y expresiones culturales.

Pero ¿qué relación hay si se entiende por competencias la forma en que una persona utiliza todos sus recursos personales (habilidades, destrezas, actitudes, conocimientos...) y el desarrollo de una educación en valores? Dar respuesta a esta pregunta no es fácil, pero lo primero de todo es que se debe partir de las siguientes premisas: la inclusión de las competencias tiene por objeto: 1) integrar los aprendizajes formales, informales y no formales; 2) poner los aprendizajes en relación con distintos tipos de contenidos, utilizándolos de manera efectiva en diferentes situaciones y contextos; y 3 ) inspirar las distintas decisiones relativas al proceso de enseñanza-aprendizaje. La primera consecuencia de todo ello es la incidencia que tiene el desarrollo de las competencias básicas en la práctica docente, y en última instancia en los centros educativos. Sin embargo, por encima de todo ello dichas competencias se plantean como un factor importante de mejora del currículo, facilitando aprendizajes socialmente válidos que los centros deben saber reflejar en los respectivos documentos institucionales y su consiguiente puesta en marcha. Por otro lado, la educación en valores, tal como se expone en la LOMCE (2013), se concreta en que

Uno de los principios en los que se inspira el Sistema Educativo Español es la transmisión y puesta en práctica de valores que favorezcan la libertad personal, la responsabilidad, la ciudadanía democrática, la solidaridad, la tolerancia, la igualdad, el respeto y la justicia, así como que ayuden a superar cualquier tipo de discriminación. Se contempla también como fin a cuya consecución se orienta el Sistema Educativo Español la preparación para el ejercicio de la ciudadanía y para la participación activa en la vida económica, social y cultural, con actitud crítica y responsable y con capacidad de adaptación a las situaciones cambiantes de la sociedad del conocimiento (Preámbulo XIV).

Así pues, el desarrollo de las competencias básicas realmente puede y debe contribuir a favorecer una educación en valores, con una proyección no sólo en el ámbito personal sino también en el social. 
Por su parte, el estudio de Zabala y Arnau (2010) destaca once ideas clave con respecto a las competencias y que se pueden sintetizar del modo siguiente:

- El uso del término competencias es una consecuencia de la necesidad de superar una enseñanza fundamentada en el aprendizaje memorístico.

- La competencia, en el ámbito educativo, ha de identificar aquello que necesita la persona para dar respuesta a los distintos problemas que se plantean a lo largo de la vida.

- Cualquier actuación competente implica el uso de conocimientos interrelacionados con habilidades y actitudes.

- Para decidir qué competencias son objeto de la educación, el paso previo es definir cuáles son sus finalidades, encaminadas al pleno desarrollo de la personalidad humana.

- Las competencias escolares deben abarcar el ámbito social, interpersonal, personal y profesional.

- El aprendizaje de una competencia implica el mayor grado de significatividad y funcionalidad posible.

- Enseñar competencias implica utilizar formas de enseñanza consistentes en dar respuesta a distintas situaciones, conflictos y problemas cercanos a la vida real.

- El análisis de las competencias debe tener en cuenta el carácter metadisciplinar de una gran parte de sus componentes.

- Una enseñanza de competencias implica la creación de un área específica para todos sus componentes de carácter metadisciplinar que incida en el aprendizaje de todas las áreas.

- Existen unas condiciones sobre cómo deben ser las estrategias metodológicas para la enseñanza de competencias que se fundamentan en el enfoque globalizador.

- Conocer el grado de dominio que el alumnado ha adquirido de una competencia es una tarea bastante compleja.

Asimismo, el estudio de Martín García y Puig Rovira (2007) señala una serie de competencias básicas para educar en valores: ser uno mismo, reconocer al otro, facilitar el diálogo, regular la participación, trabajar en equipo, hacer escuela y trabajar en red. Son competencias, que por otro lado, deben ser desarrolladas no sólo desde la escuela, sino también desde la familia y la comunidad en general. 
Como se deduce de todo ello el concepto de competencias se ha introducido con fuerza en nuestro sistema educativo, apostando por nuevas estrategias metodológicas que se supone que deben redundar en la mejora y calidad de la enseñanza y en el desarrollo integral de la persona, dentro del cual el fomento de una educación en valores se considera un aspecto imprescindible que debe estar presente tanto en la educación formal, como en la no formal e informal.

\section{La educación en valores desde ámbito educativo hasta el social}

Una de las mayores dificultades con la que se encuentran los docentes es la selección de una serie de valores y su posterior desarrollo no sólo en el ámbito educativo (que normalmente está bastante controlado), sino también fuera de dicho ámbito donde se dan una serie de condicionantes que no siempre facilitan el desarrollo e interiorización de unos valores básicos y necesarios para la formación de la persona humana. El respeto, la justicia, la responsabilidad, la libertad, la solidaridad y la autoestima, son algunos de los valores incuestionables, y como tales deben estar presentes en la vida cotidiana, sin embargo surgen algunas preguntas: ¿cómo lograr que el niño o el joven interiorice estos valores en una sociedad cada vez más compleja?..., ¿cómo asegurarnos que la práctica educativa que se lleva a cabo en nuestras escuelas e institutos es el mejor referente para la educación en estos valores dentro y fuera del ámbito escolar?..., ¿qué tipo de estrategias facilitan y garantizan esta educación en valores?... Las respuestas no son fáciles, primero hay que partir de la idea que el concepto de valor induce a distintas interpretaciones, oscilando entre la objetividad y/o subjetividad que se aplica a dicho concepto; y segundo, la educación en valores es muy compleja, precisamente por la dificultad para determinar cuáles son los valores que deben enseñarse y cómo deben enseñarse. Todo ello debe consensuarse y reflejarse en los respectivos proyectos educativos de los centros y en las programaciones didácticas, y es precisamente en la elaboración de estos documentos donde surge la primera dificultad, ya que en éstos se debe reflejar el grado de compromiso de cada uno de los miembros de la comunidad educativa, y esta implicación no siempre es fácil de delimitar.

En la práctica, la educación en valores ha venido desarrollándose con 
la LOGSE (1990) a través de los temas transversales -como contenidos curriculares- y de los contenidos actitudinales, referidos a actitudes, valores y normas, en función de unos objetivos generales de etapa; a este respecto Bolívar Botía (1995, p. 98) se mostraba contundente:

[...] la evaluación del desarrollo de actitudes y hábitos tiene que hacerse, al igual que los contenidos conceptuales, en esos ámbitos (aprendizaje de alumnos, proceso de enseñanza y programación en el Proyecto Curricular); la cuestión es qué forma diferencial y qué modos viables puede adoptar en este ámbito.

A este respecto, es evidente que los docentes están más familiarizados con la evaluación de los contenidos conceptuales, ya que la evaluación de los contenidos actitudinales y de las competencias entraña una cierta complejidad, en parte por la carga subjetiva que dichos contenidos conllevan.

Hoy, como ya se ha señalado con anterioridad los temas transversales como tal han desaparecido del currículo de forma explícita y se habla de una educación en valores como parte importante de la educación integral y armónica de la persona, afectando a las distintas etapas educativas. Pero ante esta situación, cabe preguntarnos en un principio cuáles son los factores que a priori pueden favorecer el desarrollo de los valores en el ámbito escolar: el fomento de la autoestima y la participación, la apuesta por la reflexión y el sentido crítico, o la estrecha colaboración con las familias, puede ser decisivo en una educación en valores; sin embargo, tampoco se puede obviar que la tarea es compleja y no exenta de dificultades.

\subsection{La educación en valores y su incidencia en la buena convivencia}

Reiteradamente se hace alusión a la formación integral de la persona humana en la que escuela, familia y sociedad debe contribuir a ello. El niño y el joven pasa buena parte de su tiempo en centros escolares, pero no hay la garantía de que el comportamiento desarrollado en estos centros tenga una proyección en los mismos términos al margen del centro educativo.

Debe partirse de la idea de que el conflicto forma parte de la condición humana, lo importante es saber cómo resolverlos pacíficamente 
y fomentar la buena convivencia. El conflicto como tal tiene una triple dimensión: prevención, intervención y resolución pacífica, ello supone educar para la convivencia y educar en valores. Para la resolución de conflictos, el estudio de Dudley Weeks (1998), plantea una serie de pasos a seguir:

- Crear una atmósfera efectiva.

- Clarificar percepciones.

- Apuntar a necesidades individuales y compartidas.

- Construir un poder positivo compartido.

- Mirar al futuro, luego aprender del pasado.

- Generar opciones.

- Desarrollar "factibles" los escalones para la acción.

- Hacer acuerdos mutuamente beneficiosos.

Por otro lado, el estudio de Ortega y Del Rey (2004) plantea la construcción de la convivencia basada en los siguientes aspectos:

- Constructivismo y educación de la subjetividad, aproximándose al ámbito social, afectivo y moral de la persona.

- Partir de la experiencia compartida para llegar a la reflexión personal, dentro de un contexto de diálogo.

- De la emoción al pensamiento: la reflexión compartida, de ahí la necesidad de que las experiencias personales y vivenciales puedan ser objeto de análisis.

- De la enseñanza a la educación: la importancia de la acción tutorial que favorezca la prevención de la violencia desde un buen clima de convivencia.

- La práctica: construir un modelo propio, contextualizado y elaborado y asumido por todos a través del consenso.

Como puede deducirse de ello la construcción de una convivencia pacífica supone el fomento de unos valores sociales, que impliquen los siguientes aspectos: 1) aprender a ser, logrando el mayor grado de autonomía y responsabilidad; 2) aprender a convivir, dentro del respecto y la tolerancia; y 3) aprender a convivir, llegando a ser ciudadanos activos. De ahí que sea necesario fomentar aquellos valores que propicien la resolución de conflictos dentro del marco de una buena convivencia, que en ocasiones se precisa de un proceso de mediación y/o negociación. Para ello es necesario establecer cuáles son los condicionantes que pue- 
den ayudar y/o entorpecer la resolución de un conflicto (tabla 1), entendiendo que el desarrollo de valores tales como el respeto, la tolerancia o la empatía pueden favorecer la resolución pacífica de situaciones conflictivas que se dan en la convivencia entre personas.

Tabla 1

Actitudes ante la resolución de un conflicto. Elaboración propia

\begin{tabular}{|c|c|}
\hline Ayuda & Entorpece \\
\hline Calmarse & Insultar \\
Saber escuchar & Amenazar \\
Respeto & Culpabilizar \\
Tolerancia & Acusar \\
Empatía & Despreciar \\
Diálogo & Juzgar \\
Reflexión & Ironizar \\
\hline
\end{tabular}

A este respecto, el aula puede ser un excelente medio para la resolución de conflictos dentro de la buena convivencia y en la que se hace imprescindible una educación en valores, intentado que las estrategias utilizadas tengan una proyección social más allá del propio centro educativo inclusivo. Martínez Rodríguez (2005, p. 114) defiende que:

Negociar valores significa acercar culturas, comprender las experiencias de otros, y valorar moralmente las conductas relacionadas con el espacio común. Resulta, por tanto necesario, como principio básico, abordar aquellos cuya interiorización se exige en función del modelo de sociedad y de educación que se decide colectivamente y, al mismo tiempo, aquellos otros cuya diversidad enriquece la variedad de opciones vitales, culturales y morales.

En esta misma línea, el estudio de Rodríguez Hidalgo, Zych y OrtegaRuiz (2016, p. 32) defiende una convivencia pluricultural, de tal forma que

Desde este punto de vista, la escuela debe proporcionar la oportunidad de que se aprendan valores relacionados con la inclusión y el respeto mutuo. Solo de esta manera seremos capaces de formar ciudadanos que sepan convivir en una sociedad que, en ningún caso, es homogénea, sino que por el contrario incluye esa gran diversidad en todos los sentidos. 
A la vista de todo ello, la educación en valores en la escuela inclusiva es una necesidad y es fundamental para garantizar la buena convivencia entre todos; sin embargo, a este respecto cabría preguntarse si la sociedad también es inclusiva o por el contrario en ocasiones es excluyente.

\section{A modo de conclusión}

Temas transversales, educación en valores, competencias y proyectos educativos están estrechamente relacionados. Con la LOGSE (1990) la práctica educativa tomó una nueva dimensión, y la educación deja de ser totalmente disciplinar y academicista para dar paso a una educación centrada también en valores, en actitudes que favorecen la formación integral de la persona. Aspectos que antes formaban parte del currículo oculto, en 1990 se concretan en un currículo explícito que debe ser planificado, consensuado y desarrollado por toda la comunidad educativa, aunque en realidad es el equipo docente el máximo responsable. Actualmente nuestro sistema educativo sigue apostando por una educación en valores que tenga una proyección más allá del propio centro educativo, y esto supone la colaboración de todos, no sólo de la comunidad educativa, sino también de la sociedad en general.

La institución escolar no puede permanecer al margen de las nuevas demandas sociales, y ya su función no es sólo instruir o transmitir unos conocimientos, sino que sus competencias se han ampliado, y ahora debe formar personas en el pleno sentido de la palabra. Debe desarrollarse un proceso de humanización que implique una educación en valores, es decir, que eduque personas libres, tolerantes, responsables, solidarias, etc., a través de unos modelos educativos válidos y del desarrollo de una serie de contenidos actitudinales y transversales que contribuyan precisamente a formar personas, o mejor dicho a

[...] formar el carácter, en el sentido más extenso y total del término: formar el carácter para que se cumpla un proceso de socialización imprescindible, y formarlo para promover un mundo más civilizado, crítico con los defectos del presente y comprometido con el proceso moral de las estructuras y actitudes sociales (Camps Cervera, 1996, p. 11). 
Todos los educadores se deben implicar en una educación de valores, que garantice la formación plena de las personas, en todos sus ámbitos, y en función de ello organizar toda una propuesta curricular y educativa abierta y plural. En definitiva, una actuación conjunta para favorecer el desarrollo integral y armónico de la persona, a través de una convivencia pacífica, y desarrollando una educación en valores. Asimismo, este planteamiento debe incardinarse dentro de la escuela inclusiva en la que todos tienen cabida con sus intereses y necesidades individuales. En este sentido, el estudio de Rosales (2015, p. 46) entiende que

La creación de un clima satisfactorio, en las aulas y los centros escolares, constituye un punto de partida básico, para el desarrollo de una enseñanza caracterizada por la equidad y la inclusión. Este clima afecta a la convivencia en las aulas y fuera de ellas, a las relaciones entre alumnos y profesores, entre éstos y los padres y madres, también a las relaciones entre alumnos y entre profesores, y se puede entender en los ámbitos de actuación formal y no formal o informal.

Por otra parte, el Informe Delors (1996) basa la educación en cuatro pilares: aprender a conocer, aprender a hacer, aprender a vivir juntos y aprender a ser, y es precisamente en estos dos últimos pilares en donde la educación en valores adquiere un protagonismo especial: aprender a vivir en sociedad supone la comprensión del otro y prepararse para tratar los conflictos, respetando los valores de pluralismo (muy vinculado a la escuela inclusiva), comprensión mutua y paz; por su parte, aprender a ser implica desarrollar una capacidad de autonomía, de juicio y responsabilidad personal. Como puede deducirse de dicho Informe, los valores sociales constituyen unos de los rasgos definitorios de la educación del presente y el futuro.

Asimismo, como ya se ha señalado con anterioridad, el desarrollo de las competencias recogidas en el actual sistema educativo es otro de los factores que también contribuye a la educación en valores, sobre todo en lo que se refiere a las competencias sociales y cívicas, haciendo referencia a las capacidades para relacionarse con las personas y participar de manera activa, participativa y democrática en la vida social y cívica.

La educación en valores debe contemplarse con claridad en los proyectos educativos de los centros, tomando como referencia las características socioculturales del alumnado y el entorno en el que se encuentra 
ubicado el centro, estableciendo las responsabilidades de cada colectivo (familias, profesorado, alumnado) y utilizando todos aquellos recursos que favorezcan una formación en valores a nivel personal y social. El proyecto educativo de centro debe ser el referente para los planes de convivencia, y las normas de organización, funcionamiento y convivencia del centro.

Tomando como referencia todo lo anteriormente expuesto, se considera que hay una serie de estrategias concretas que pueden favorecer la educación en valores dentro de la escuela inclusiva, destacando entre todas las siguientes: el diálogo, tener tiempo para hablar y escuchar, establecer normas claras y consensuadas, aprender a resolver conflictos de forma pacífica sin violencia, permitir expresar sentimientos en el aula, corregir sin dejar de mostrar ternura, saber pedir disculpas cuando nos equivocamos, mediar cuando sea necesario, ser coherentes, etc. No obstante, lo importante es que estas estrategias, primeramente, cuenten con la colaboración de la familia y luego tenga una proyección más allá del ámbito escolar y familiar que se consolide a lo largo del desarrollo integral de la persona.

\section{Referencias}

Bisquerra, R. (2011). Diversidad y escuela inclusiva desde la educación emocional. Recuperado de: http://diversidad.murciaeduca.es/publicaciones/diversa2011/docs/bisquerra.pdf

Bolívar Botía, A. (1995). La evaluación de valores y actitudes. Madrid: Anaya- Alauda.

Buxarrais, M.R. (2003). La formación del profesorado en la educación en valores. Propuesta y materiales. Bilbao: Desclée De Brouwer.

Camps Cervera, V. (1996). Los valores de la educación. Madrid: Anaya- Alauda.

Del Carmen, L. (2004). La atención a la diversidad: una cuestión de valores. En AA.VV. La escuela inclusiva. Prácticas y reflexiones. (47-55). Barcelona: Graó.

Delors, J. (Coord.) (1996). La educación encierra un tesoro. Comisión Internacional sobre la Educación para el siglo XXI. Madrid: Santillana/UNESCO.

Domínguez Chillón, G. (1999). Los valores en Educación Infantil. Madrid: La Muralla.

Dudley Weeks, Ph. D. (1998). 8 Pasos para resolver conflictos. Buenos Aires: Editor, S.A. Javier Vergara.

González Lucini, F. (1994). Temas Transversales y Educación en Valores. Madrid: AnayaAlauda.

López Melero, M. (2012). La escuela inclusiva: una oportunidad para humanizarnos. Revista Interuniversitaria de Formación del Profesorado, 74 (26,2), 131-160.

Martín García, X. y Puig Rovira, J.M. (2007). Las siete competencias básicas para educar en valores. Barcelona: Graó. 
Evolución de la educación en valores y su proyección social en la escuela inclusiva Rosa Ma Santamaría Conde y Miguel Corbí Santamaría

Martínez Rodríguez, J.B. (2005). Educación para la ciudadanía. Madrid: Morata.

Ministerio de Educación y Ciencia (1993). Temas Transversales y Desarrollo Curricular. Madrid: Secretaría de Estado de Educación.

Muntaner Guasp, J.J., Rosselló Ramón, M.R. y De la Iglesia Mayol, B. (2016). Buenas prácticas en educación inclusiva. Educatio Siglo XXI, 34 (1), 31-50.

Ortega, R. y Del Rey, R. (2004). Construir la convivencia. Barcelona: Edebé.

Rodríguez Hidalgo, A.J., Zych, I. y Ortega-Ruiz, R. (2016). El centro y el aula como escenarios de la convivencia. En Ortega-Ruiz, R. y Zych, I. (eds.). Convivencia escolar. Manual para docentes. (27-37). Madrid: Grupo 5.

Rosales, C. (2015). Los temas transversales en el aula. Convivencia, salud e igualdad. Santiago de Compostela (A Coruña): Andavira editora.

Yus Ramos, R. (2002). Temas transversales y educación en valores: la educación del siglo XXI. En AA.VV. Valores escolares y educación para la ciudadanía. (33-44). Barcelona: Graó.

Zabala, A. y Arnau, L. (2010). 11 Ideas clave. Cómo aprender y enseñar competencias. Barcelona: Graó. 
